\title{
Topical issue on the tower of effective (field) theories and the emergence of nuclear phenomena
}

\author{
Vincent Bontems ${ }^{1}$, Thomas Duguet ${ }^{1,2, a}$, Gaute Hagen ${ }^{3,4}$, Vittorio Somà ${ }^{1}$ \\ ${ }^{1}$ IRFU, CEA, Université Paris-Saclay, 91191 Gif-sur-Yvette, France \\ ${ }^{2}$ KU Leuven, Instituut voor Kern- en Stralingsfysica, 3001 Leuven, Belgium \\ ${ }^{3}$ Physics Division, Oak Ridge National Laboratory, Oak Ridge, TN 37831, USA \\ ${ }^{4}$ Department of Physics and Astronomy, University of Tennessee, Knoxville, TN 37996, USA
}

Published online: 29 January 2021

(C) The Author(s), under exclusive licence to Società Italiana di Fisica and Springer-Verlag GmbH Germany, part of Springer Nature 2021

Throughout the twentieth century, the astonishingly rich phenomenology nuclear systems display at low energy has led theorists to develop a large mosaic of nuclear models. This variety of models makes however difficult to elucidate the deeper ambitions of this research activity given that epistemological tools have been rather elaborated to account for a unified and stabilized theory than to apprehend a plurality of models. Indeed, the scientific value of a theory is typically evaluated in terms of the precision of its prediction, of its range of applicability and of the intelligibility of its principles. Ideally, a theory is thus meant to be reductionist, unifying and fundamentalist. In view of the intrinsic limited precision of their prediction and of the difficulty to assess a priori their range of applicability, as well as of their specific and disconnected character, traditional models of inter-nucleon interactions and of nuclear structure and reactions are necessarily deficient when analyzed by means of standard epistemological interpretative frameworks. In this context, the main benefit of the notions of emergence and effectiveness is to offer the possibility of a more pertinent reformulation of the key questions at play.

The notion of emergence first arose in connection with a philosophical reflection on the relationship a material substrate entertains with properties of an organism that seem to be irreducible to this substrate: for example, how life emerges from matter (in a vitalist perspective) or how conscience emerges from brain's activity (in an idealist perspective). Later on, the notion disseminated into intra-disciplinary debates on the modeling of complex physical or biological systems: emergence steps in whenever properties observed at a certain scale are inexplicable or unpredictable starting from an underlying scale. As such, it challenges the understanding and the articulation of the various organizational

a e-mail: thomas.duguet@cea.fr (corresponding author) levels of complex systems. The emergence problem reactivates the ancient debate between partisans of reductionism and holism, the latter considering after Aristotle that properties of an organism as a whole cannot be identified to those resulting from the aggregation of its parts.

The notion of effective reality, which distinguishes an actuality from the vague notion of "reality", was also first elaborated by the philosophical reflection. In Science of Logic, Hegel instituted the effective reality (Wirklichkeit) as a primordial category to underline the fact that only "what is effective can act": effective reality is a law that first imposes itself onto reality as an arbitrary necessity whose justification can only be captured a posteriori by human's mind in that its activity is precisely what reveals the most significant aspects of reality. Independently of this elaboration, scientific language seized this notion to designate theories relying on a deliberate rejection of the explicative factors originating from the underlying scales. It constitutes a strategy that opposes the reductionist ambition when the knowledge of the explicative factors from the underlying scales defaults or when their complexity makes predictions impractical. Even more so, the use of an effective theory can be justified when the reductionist view is at reach but unnecessarily complicated to apprehend the phenomena of interest. Emergence and effectiveness embody two symmetric ruptures with respect to the standard set by reductionist, unifying and fundamentalist theories: while emergence signals the occurrence of phenomena that challenge the ambition to explain everything starting from a fundamental scale, effectiveness notifies that it might actually be preferable to escape such a demand. Furthermore, emergence and effectiveness define two alternative viewpoints regarding what a theory must be. The former institutes a tension with the reductionist explanation that encourage scientific rationalism to deepen its comprehension of the relative character of the various 
levels of organization whereas effective theories arrogate a pragmatic justification.

Following a revision of the meaning of renormalization procedures in particle physics, Steven Weinberg rehabilitated and promoted the notion of effective field theories, before initiating chiral effective field theory that has been paving the way for a reconfiguration of low-energy nuclear physics. The development of effective theories (and their interlocking) represents a considerable transformation compared to the situation embodied by the plurality of models: a new exigence of systematicity implying the necessity

1. to state a priori the range of applicability of the theory and to specify the pertinent degrees of freedom, along with the symmetries originating from the underlying scales,

2. to motivate (often on the basis of "naturalness") an organization (a "power counting") of all interaction operators allowed by symmetries,

3. to determine the low-energy constants associated with the unresolved physics, either deductively from the underlying effective theory or inductively from experiment.

While the portfolio of nuclear models constitutes a compromise between the necessity to account for emerging phenomena and the demand for a reductionist explanation, compromise whose status remains the one of a norm by default, effective theories break away from the inferiority complex with respect to the "grand theory". They incarnate themselves through the coherence of the boundaries they assess to their own applicability and through the selection of the underlying factors they explicitly retain. The intriguing zoology of nuclear models now extends into the research, yet labyrinthine, of a hierarchy or a tree view of nuclear effective theories paving the composite rationality of the nuclear domain. However, it would be presumptuous to believe having solved, or even anticipated, all the obstacles by the sole transitioning from the perspective of models to the perspective of effective theories. Contrarily, an entire range of new thorny questions arises and indicates, paradoxically, the fact that it is impossible to give neither the surprises of emergence nor the frustration of rationalism their final notice.

Indeed, the first successes of effective theories in the context of low-energy nuclear science unavoidably question the frontiers of complexity, the naturalness of scales separation between effective theories and the limit of a theoretical construct via a bottom-up strategy. The issue associated with the articulation of a potential arborescence of nuclear effective theories is accompanied by the uncertainty of the criteria supposed to order each of them: should one invoke systematicity, naturalness or commodity? Besides, unexpected emerging phenomena/scales can indicate that the power counting organizing the hierarchy of operators in such or such effective theory may not be optimal anymore. In this context, epistemological issues are not as massive and abstract as metaphysical questions are, i.e. "does nature display a bottom?" or "do our theories possess a double-bottom?", but rather relate to fine-tuning procedures. The fact remains that it is by maintaining the fertile tension between the rationalist ambition to make the range of applicability intelligible and the pragmatist adjustment of the theoretical tools that one can hope to clarify the unanswered questions at play in low-energy nuclear physics.

While being far from covering all relevant points, the present Topical Issue aims at addressing and clarifying some of the points elaborated on above with the ambition to help articulating a penetrating path forward for low-energy nuclear physics. The issue contains two complementary parts. The first part is dedicated to discussing the philosophical foundations of effective field theories before introducing how these notions come into play in biological, gravitational and low-energy nuclear systems. The second part of the Topical Issue then contains contributions focusing on state-of-the art developments in nuclear theory. The objective is to discuss recent advances and try to systematically address the thorny questions that have or may arise.

V. Bontems, T. Duguet, G. Hagen, V. Somà

Guest Editors

\section{Table of contents}

\section{Reductionism, emergence and effective theories}

(a) Philosophical Foundations of Effective Field Theories (S. Rivat, A. Grinbaum) [1]

(b) Progress in Integrative Systems Biology, Physiology and Medicine: Towards a Scale Relative Biology (C. Auffray, D. Noble, L. Nottale, P. Turner) [2]

(c) Gravitons and pions (J. F. Donoghue) [3]

(d) Naturalness in Nuclear Effective Field Theories (U. van Kolck) [4]

\section{From the era of nuclear models to the era of nuclear effective (field) theories?}

(a) An update on fine-tunings in the triple-alpha process (T. A. Lähde, U.-G. Meißner, E. Epelbaum) [5]

(b) Can chiral EFT give us satisfaction? (R. Machleidt, F. Sammarruca) [6]

(c) A Consistency Test of EFT Power Countings from Residual Cutoff Dependence (H. W. Grießhammer) [7]

(d) Uncertainty quantification and falsification of Chiral Nuclear Potentials (R. Navarro Pérez, E. Ruiz Arriola) [8] 
(e) Do we know how to count powers in pion-less and pion-full effective field theory? (C.-J. Yang) [9]

(f) Towards high-order calculations of three-nucleon scattering in chiral effective field theory (E. Epelbaum, J. Golak, K. Hebeler, H. Kamada, H. Krebs, U.-G. Meißner, A. Nogga, P. Reinert, R. Skibiǹski, K. Topolnicki, Y. Volkotrub, and H. Witała) [10]

(g) Hyperon-nucleon interaction within chiral effective field theory revisited (J. Haidenbauer, U.-G. Meißner, A. Nogga) [11]

(h) Heavy hadron molecules in effective field theory: the emergence of exotic nuclear landscapes (M. Pavon Valderrama) [12]

(i) Cluster effective field theory and nuclear reactions (S.-I. Ando) [13]

(j) Energies and radii of light nuclei around unitarity (S. König) [14]

(k) Emergence of simple patterns in many-body systems: from macroscopic objects to the atomic nucleus (R.F. Garcia Ruiz, A. Vernon) [15]

(1) Renormalization of pionless effective field theory in the A-body sector (M. Drissi, T. Duguet, and V. Somà) [16]

(m) Probing ab initio emergence of nuclear rotation (Mark A. Caprio, P. J. Fasano, P. Maris, A. E. McCoy, J. P. Vary) [17]

(n) Neutron matter at the interface(s) - Static response and effective mass (M. Buraczynski, N. Ismail, A. Gezerlis) [18]

(o) Turning the nuclear energy density functional method into a proper effective field theory: reflections (R. J. Furnstahl) [19].

\section{References}

1. S. Rivat, A. Grinbaum, Eur. Phys. J. A 56, 90 (2020). https://doi. org/10.1140/epja/s10050-020-00089-w

2. C. Auffray, D. Noble, L. Nottale, P. Turner, Eur. Phys. J. A 56, 88 (2020). https://doi.org/10.1140/epja/s10050-020-00090-3

3. J.F. Donoghue, Eur. Phys. J. A 56, 86 (2020). https://doi.org/10. 1140/epja/s10050-020-00091-2

4. U. van Kolck, Eur. Phys. J. A 56, 97 (2020). https://doi.org/10. 1140/epja/s10050-020-00092-1

5. T.A. Lähde, U.-G. Meißner, E. Epelbaum, Eur. Phys. J. A 56, 89 (2020). https://doi.org/10.1140/epja/s10050-020-00093-0

6. R. Machleidt, F. Sammarruca, Eur. Phys. J. A 56, 95 (2020). https:// doi.org/10.1140/epja/s10050-020-00101-3

7. H.W. Grießhammer, Eur. Phys. J. A 56, 118 (2020). https://doi.org/ 10.1140/epja/s10050-020-00129-5

8. R. Navarro Pérez, E. Ruiz Arriola, Eur. Phys. J. A 56, 99 (2020). https://doi.org/10.1140/epja/s10050-020-00103-1

9. C.-J. Yang, Eur. Phys. J. A 56, 96 (2020). https://doi.org/10.1140/ epja/s10050-020-00104-0

10. E. Epelbaum, J. Golak, K. Hebeler, H. Kamada, H. Krebs, U.-G. Meißner, A. Nogga, P. Reinert, R. Skibinski, K. Topolnicki, Y. Volkotrub, H. Witała, Eur. Phys. J. A 56, 92 (2020). https://doi. org/10.1140/epja/s10050-020-00102-2

11. J. Haidenbauer, U.-G. Meißner, A. Nogga, Eur. Phys. J. A 56, 91 (2020). https://doi.org/10.1140/epja/s10050-020-00100-4

12. M. Pavon Valderrama, Eur. Phys. J. A 56, 109 (2020). https://doi. org/10.1140/epja/s10050-020-00099-8

13. S.-I. Ando, Eur. Phys. J. A 57, 17 (2021). https://doi.org/10.1140/ epja/s10050-020-00304-8

14. S. König, Eur. Phys. J. A 56, 113 (2020). https://doi.org/10.1140/ epja/s10050-020-00098-9

15. R.F. Garcia Ruiz, A. Vernon, Eur. Phys. J. A 56, 136 (2020). https:// doi.org/10.1140/epja/s10050-020-00134-8

16. M. Drissi, T. Duguet, V. Somà, Eur. Phys. J. A 56, 119 (2020). https://doi.org/10.1140/epja/s10050-020-00097-w

17. M.A. Caprio, P.J. Fasano, P. Maris, A.E. McCoy, J.P. Vary, Eur. Phys. J. A 56, 120 (2020). https://doi.org/10.1140/epja/ s10050-020-00112-0

18. M. Buraczynski, N. Ismail, A. Gezerlis, Eur. Phys. J. A 56, 112 (2020). https://doi.org/10.1140/epja/s10050-020-00096-2-X

19. R.J. Furnstahl, Eur. Phys. J. A 56, 85 (2020). https://doi.org/10. 1140/epja/s10050-020-00095-y 